\title{
Erratum to: Selection Response Decomposition (SRD): A New Tool for Dissecting Differences and Similarities Between Matrices
}

\author{
Gabriel Marroig • Diogo Melo • Arthur Porto • \\ Harley Sebastião • Guilherme Garcia
}

Published online: 6 July 2012

(C) Springer Science+Business Media, LLC 2012

\section{Erratum to: Evol Biol (2011) 38:225-241 \\ DOI 10.1007/s11692-010-9107-2}

It has come to our attention that the article 'Selection Response Decomposition (SRD): A New Tool for Dissecting Differences and Similarities Between Matrices' (Marroig et al. 2011) contains an error in Fig. 1. This figure should show the mean and confidence intervals for the correlation between random vectors in various dimensions. We take this opportunity to correct the error, providing the correct figure, and to make a few comments on the null expectation for the correlation between random vectors. This null hypothesis is used in both the Random Skewers and the Selection Response Decomposition techniques described in our manuscript.

The first relevant point is the option of taking or not the absolute value of the correlation between the random vectors. This depends on the question being asked. Taking the

The online version of the original article can be found under doi:10.1007/s11692-010-9107-2.

G. Marroig ( $\varangle)$ · D. Melo · A. Porto · H. Sebastião · G. Garcia Laboratório de Evolução de Mamíferos, Departamento de Genética e Biologia Evolutiva, Instituto de Biociências, Universidade de São Paulo, CP 11461, CEP 05508-090 São Paulo, SP, Brazil

e-mail: gmarroig@usp.br

A. Porto

Evolution, Ecology and Population Biology Program, Department of Biology, Washington University in St Louis, St Louis, MO, USA

Present Address:

G. Marroig

Rua do Matão 277, sala 300, CEP 05508-090 Butantã,

São Paulo, Brazil absolute value of the correlation implies that we are only interested in the relationship between a vector and a particular line through the origin in the vector space, without considering whether the vector is in the 'positive' or 'negative' side of this line. This would be the case when comparing principal components (whose sign is arbitrary) or lines of least evolutionary resistance (Schluter 1996). The null expectation for this case is shown in Fig. 1a, along with appropriate confidence intervals, obtained by simulating 100,000 random vectors drawn from a spherically symmetrical distribution and noting their correlation with a fixed random vector.

The other possibility is simply not to take the absolute value of the correlation between random vectors. This implies that the direction is relevant, for instance when comparing particular modifications on a set of characters between two species or comparing two matrices. This null expectation can be seen in Fig. $1 b$.

In spite of these differences, the significance cut-off for both methods is very similar, and even if one is used instead of the other, interpretation of the results should remain largely unchanged. 


\section{a}

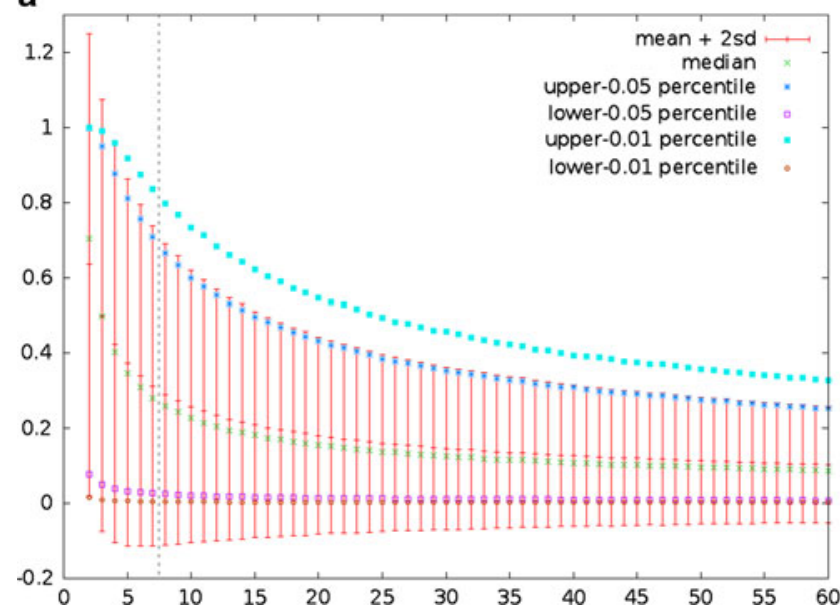

Fig. 1 a The absolute vector correlation average, median, average $\pm 2 x$ standard deviation and the 95 and $99 \%$ upper and lower empirically observed percentiles based on random vectors correlation with increasing dimensionality (from $n=2$ to $n=60$ ). The patterned line indicates our suggestion for the lower dimensionality

\section{References}

Marroig, G., Melo, D., Porto, A., Sebastião, H., \& Garcia, G. (2011). Selection response decomposition (SRD): A new tool for b

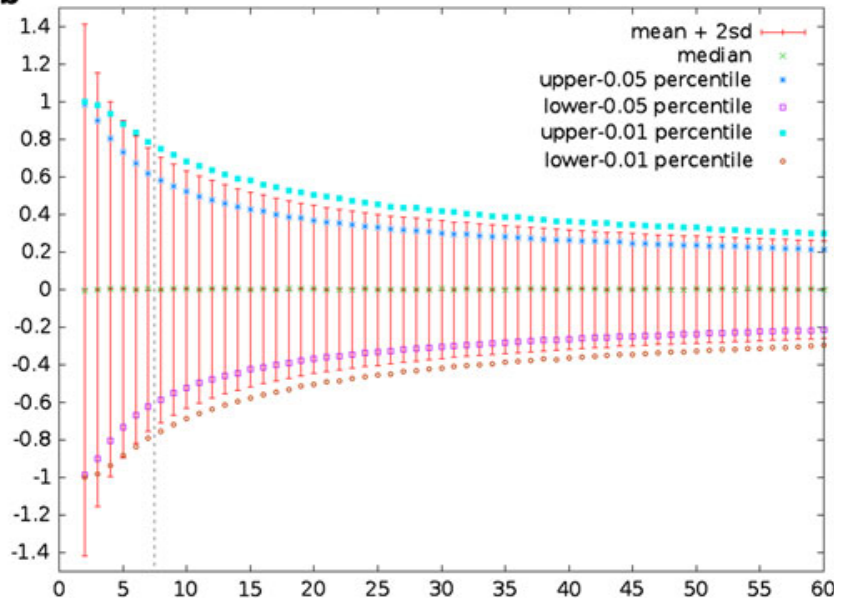

where the SRD and the RS methods based on vector correlations can be used; $\mathbf{b}$ the same statistics as in $\mathbf{a}$ (average, median, etc.) presented for the random vector correlation without taking the absolute values, as usually, will be the case for the SRD

dissecting differences and similarities between matrices. Evolutionary Biology, 38(2), 225-241.

Schluter, D. (1996). Adaptive radiation along genetic lines of least resistance. Evolution, 50(5), 1766-1774. 